\title{
K2 DISCOVERS A BUSY BEE: AN UNUSUAL TRANSITING NEPTUNE FOUND IN THE BEEHIVE CLUSTER
}

\author{
Christian Obermeier $^{1,2,3}$, Thomas Henning $^{1}$, Joshua E. Schlieder ${ }^{4,5,16}$, Ian J. M. Crossfield ${ }^{6,17}$, Erik A. Petigura ${ }^{7,18}$, \\ Andrew W. Howard ${ }^{8}$, Evan SinukofF ${ }^{8,19}$, Howard IsaAcson ${ }^{9}$, David R. Ciardi ${ }^{5}$, Trevor J. David ${ }^{10}$, \\ Lynne A. Hillenbrand ${ }^{10}$, Charles A. Beichman ${ }^{9}$, Steve B. Howell ${ }^{4}$, Elliott Horch ${ }^{11}$, Mark Everett ${ }^{12}$, Lea Hirsch ${ }^{9}$, \\ Johanna Teske ${ }^{13,16}$, Jessie L. Christiansen ${ }^{5}$, Sébastien Lépine ${ }^{14}$, Kimberly M. Aller ${ }^{8}$, Michael C. LiU ${ }^{8}$, \\ Roberto P. Saglia ${ }^{2}$, John Livingston ${ }^{15}$, and Matthias Kluge ${ }^{2,3}$ \\ Max Planck Institut für Astronomie, Heidelberg, Germany \\ ${ }^{2}$ Max-Planck-Institute for Extraterrestrial Physics, Garching, Germany \\ ${ }^{3}$ University Observatory Munich (USM), Ludwig-Maximilians-Universität, Munich, Germany \\ ${ }^{4}$ NASA Ames Research Center, Moffett Field, CA, 91125, USA \\ 5 NASA Exoplanet Science Institute, California Institute of Technology, Pasadena, CA, 91125, USA \\ ${ }^{6}$ Lunar \& Planetary Laboratory, University of Arizona, 1629 E. University Blvd., Tucson, AZ, 85721, USA \\ ${ }^{7}$ Geological and Planetary Sciences, California Institute of Technology, Pasadena, CA, 91125, USA \\ ${ }^{8}$ Institute for Astronomy, University of Hawai' ${ }^{i}$ at Mānoa, Honolulu, HI, 96822, USA \\ ${ }^{9}$ Astronomy Department, University of California, Berkeley, CA, 94720, USA
${ }^{10}$ Department of Astronomy, California Institute of Technology, Pasadena, CA, 91125, USA \\ ${ }^{11}$ Department of Physics, Southern Connecticut State University, New Haven, CT, 06515, USA \\ ${ }^{12}$ National Optical Astronomy Observatory, Tucson, AZ, 85719, USA \\ ${ }^{13}$ Carnegie Department of Terrestrial Magnetism, Washington, DC, 20015, USA \\ ${ }^{14}$ Department of Physics and Astronomy, Georgia State University, GA, USA \\ ${ }^{15}$ Department of Astronomy, Graduate School of Science, The University of Tokyo, 7-3-1 Bunkyo-ku, Tokyo 113-0033, Japan \\ Received 2016 May 16; revised 2016 July 20; accepted 2016 August 3; published 2016 December 6
}

\begin{abstract}
Open clusters have been the focus of several exoplanet surveys, but only a few planets have so far been discovered. The Kepler spacecraft revealed an abundance of small planets around small cool stars, therefore, such cluster members are prime targets for exoplanet transit searches. Kepler's new mission, K2, is targeting several open clusters and star-forming regions around the ecliptic to search for transiting planets around their low-mass constituents. Here, we report the discovery of the first transiting planet in the intermediate-age (800 Myr) Beehive cluster (Praesepe). K2-95 is a faint $(K \mathrm{p}=15.5 \mathrm{mag}) \mathrm{M} 3.0 \pm 0.5$ dwarf from $K 2$ 's Campaign 5 with an effective temperature of $3471 \pm 124 \mathrm{~K}$, approximately solar metallicity and a radius of $0.402 \pm 0.050 R_{\odot}$. We detected a transiting planet with a radius of $3.47_{-0.53}^{+0.78} R_{\oplus}$ and an orbital period of 10.134 days. We combined photometry, medium/high-resolution spectroscopy, adaptive optics/speckle imaging, and archival survey images to rule out any false-positive detection scenarios, validate the planet, and further characterize the system. The planet's radius is very unusual as M-dwarf field stars rarely have Neptune-sized transiting planets. The comparatively large radius of K2-95b is consistent with the other recently discovered cluster planets K2-25b (Hyades) and K2-33b (Upper Scorpius), indicating systematic differences in their evolutionary states or formation. These discoveries from $K 2$ provide a snapshot of planet formation and evolution in cluster environments and thus make excellent laboratories to test differences between field-star and cluster planet populations.
\end{abstract}

Key words: eclipses - stars: individual (K2-95) - stars: low-mass - techniques: photometric - techniques: spectroscopic

\section{INTRODUCTION}

Exoplanet science is still a young field, but what stands out is the strong diversity in the properties of both detected planets and their host stars. Already a short time after the first transiting planet was detected by Charbonneau et al. (2000) and Henry et al. (2000), surveys were started with a focus on open clusters for a variety of reasons. The higher density of stars gives surveys access to more stars for a given field of view. Age, distance, and metallicity of the member stars are well determined, yielding more precise estimates for the planetary

\footnotetext{
16 NASA Postdoctoral Program Fellow.

17 NASA Sagan Fellow.

18 Hubble Fellow.

19 NSERC Postgraduate Research Fellow.

${ }^{20}$ Carnegie Origins Postdoctoral Fellow, jointly appointed by Carnegie DTM and Carnegie Observations.
}

and stellar parameters. Furthermore, most observed field stars are relatively old $(\geqslant 1 \mathrm{Gyr})$ while many currently targeted clusters present a younger sample (10-800 Myr). In addition, planet formation in stellar clusters may well be very different due to stronger and more frequent gravitational interactions between the stars. Planets in younger clusters may also be undergoing thermal evolution, radial contraction, or receiving high irradiation from their active host stars. Therefore, open clusters are an excellent laboratory to test planet formation and evolution models. Initial transit surveys that focused on 47 Tuc (Gilliland et al. 2000; Weldrake et al. 2005), NGC 2301 (Howell et al. 2005) and NGC 7789 (Bramich \& Horne 2006), found no evidence for transiting planets. Since then, fourteen planets have been discovered in open clusters, namely in NGC 6811 (Meibom et al. 2013), NGC 2423 (Lovis \& Mayor 2007), M67 (Brucalassi et al. 2014, 2016), the Beehive (Praesepe) (Quinn et al. 2012; Malavolta et al. 2016), the Hyades (Sato 
et al. 2007; Quinn et al. 2014; David et al. 2016a; Mann et al. 2016a) and Upper Scorpius (David et al. 2016b; Mann et al. 2016b). All planets in M67, the planet in NGC 2423, one planet in the Hyades and the Praesepe planets were detected with the radial velocity (RV) method. All planets in NGC 6811, one planet in the Hyades and the planet in Upper Scorpius were discovered with the transit method. All detections were of planets that likely harbor significant gaseous envelopes. Additionally, a 2 Myr old hot Jupiter located in the TaurusAuriga star-forming region was detected via the RV method (Donati et al. 2016).

All transiting planets were detected with the Kepler space telescope. After the failure of two of its four reaction wheels, the original mission of Kepler ended and was redirected for the "second light" survey K2 (Howell et al. 2014). Instead of continuously observing the same area over years, the $K 2$ mission switches fields every three months, stabilized by the two remaining reaction wheels and solar photon pressure for the third axis (roll angle). However, the telescope still drifts slowly and has to be corrected by firing the thrusters every $6 \mathrm{hr}$. Photometric precision is therefore slightly lower than during the Kepler mission but, as will be described in the following section, can be corrected very well.

The Beehive cluster (M44), also called Praesepe, is an open cluster targeted by $K 2$ in Campaign 5 . It is nearby $(d=183 \pm 8 \mathrm{pc}$, van Leeuwen 2009; Majaess et al. 2011) and of intermediate age. Past estimates placed the age of Praesepe at around $600 \mathrm{Myr}$ (Fossati et al. 2008) but new estimates that take into account the effects of rotation in its high-mass members suggest an age as old $800 \mathrm{Myr}$ (Brandt \& Huang 2015b). Furthermore, the kinematics (Madsen et al. 2002), metallicity (Dobbie et al. 2006) and age (Brandt \& Huang 2015b) of Praesepe are very similar to those of the Hyades cluster. The age of Hyades was also redetermined to 800 Myr (Brandt \& Huang 2015a; David \& Hillenbrand 2015) and it is now assumed that both clusters may share the same origin.

Since the transit signal gets stronger with decreasing stellar radius, $\mathbf{M}$ dwarfs are promising targets for the detection of small planets in an open cluster. Dressing \& Charbonneau (2015) estimate an abundance of rocky and small subNeptunian planets around those stars with periods shorter than 200 days with an average of $2.5 \pm 0.2$ planets per star with radii between 1 and $4 R_{\oplus}$. Here, we present the discovery and validation of a transiting Neptune-sized planet in the Praesepe cluster detected in $K 2$ Campaign 5 in orbit around the low-mass star K2-95. In Section 2 we describe the layout of our photometric and spectroscopic follow-up and detail the subsequent results in Section 3. We validate the candidate as a planet in Section 4, discuss the impact of our findings in the context of exoplanets in clusters and the field in Section 5, and provide concluding remarks in Section 6.

\section{OBSERVATIONS}

\subsection{K2 Target Selection and Photometry}

We identified the star K2-95 as a potential M dwarf target and high probability member of the Praesepe cluster for our $K 2$ Campaign 5 proposal (GO5006-PI Schlieder). Other groups also proposed this star as a potential K2 target (GO5011PI Beichman, GO5048-PI Guzik, GO5095-PI Agueros, GO5097-PI Johnson).
K2-95 was observed during K2 Campaign 5 with nearly continuous photometry from 2015 April 27 to July 10. We extracted the photometry from the pixel data which we downloaded from the MAST. ${ }^{21}$

Our photometric extraction pipeline is described in more detail in Petigura et al. (2015) and Crossfield et al. (2015). During $K 2$ operations, the telescope is torqued by solar radiation pressure which causes it to slowly roll around the boresight. This motion causes stars to drift across the CCD by about 1 pixel every $6 \mathrm{hr}$. As stars are sampled by different pixels, intra-pixel sensitivity and flat-fielding variations cause the apparent brightness of the star to change. Thruster fires to correct for this drift affect the pointing and therefore pixel position greatly, giving the overall photometry a saw-tooth shape. We solve for the roll angle between each frame and an arbitrary reference frame and model the time- and rolldependent brightness variations using a Gaussian process. Further, we adjust the size of our square extraction aperture to minimize the residual noise in the corrected light curve. This balances two competing effects: larger apertures yield smaller systematic errors while smaller apertures include less background noise. Our final square extraction aperture is $r=1$ pixel $\approx 4^{\prime \prime}$. The resulting, de-trended light curve exhibits slow, periodic, $\sim 1 \%$ modulations with a period of about 24 days. We attribute this modulation to spots on the rotating stellar surface. The timescale of this variation is long compared to other $\mathrm{M}$ dwarfs in Praesepe and places K2-95 among the slowest rotators in the cluster (see also Section 3.5). This variation is fitted and removed to produce the final light curve which is shown in the top panel of Figure 1.

We searched through the optimized light curve with the TERRA algorithm which is described in more detail by Petigura et al. (2013). In short, it searches for periodic boxshaped photometric dimmings and fits them with a model from Mandel \& Agol (2002). Using TERRA, we detected a transit signal in the K2-95 light curve with a period of $P=10.132$ days and a signal-to-noise ratio $(\mathrm{S} / \mathrm{N})$ of 23.97 . The phase-folded light curve is shown in the bottom panel of Figure 1, centered around the transit event. We subtracted the best-fitting model transit and iterated the TERRA algorithm to search for other transits but did not detect any secondary signals. Visual inspection also did not reveal any additional transit features.

\subsection{Photometric Follow-up}

We observed K2-95 with the $2.0 \mathrm{~m}$ Fraunhofer telescope Wendelstein (Hopp et al. 2014), using the Wide Field Imager (WFI) (Kosyra et al. 2014) on Mt. Wendelstein in the Bavarian Alps. An independent transit detection from a ground-based facility serves not only for period confirmation and estimation of its uncertainty, but as evidence for the planetary nature of the transit from a common eclipse depth at different wavelengths. Multi-band transit photometry can be used to characterize the planet's atmosphere or rule out false-positive detections (Mislis et al. 2010; Southworth et al. 2012; Mancini et al. 2013; Ciceri et al. 2016). The limb darkening coefficients differ across photometric bands and can be used to differentiate between planetary signals and those of shallow-eclipse eclipsing binaries (EBs). K2-95 was followed up in the $i^{\prime}$-band on UT 2016 April 16 during suboptimal weather with seeing between

\footnotetext{
21 The Mikulski Archive for Space Telescopes.
} 

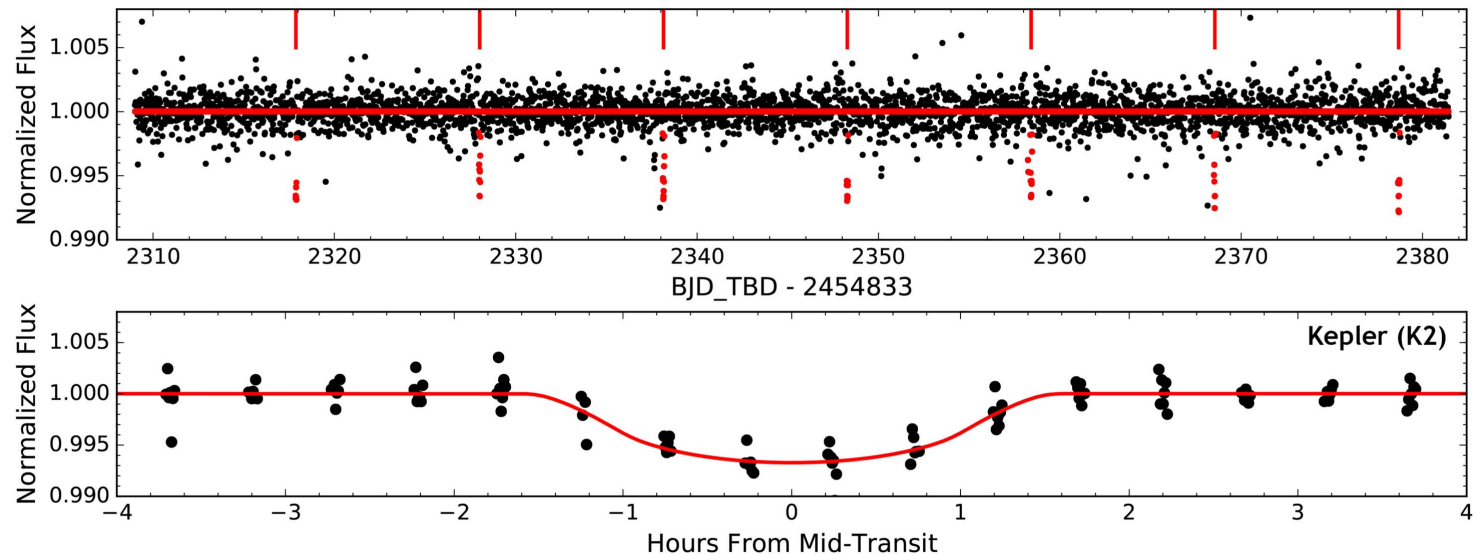

Figure 1. Top: calibrated and normalized $K 2$ photometry for K2-95. The upper red lines indicate the detected transits with the corresponding points also marked in red. Bottom: period-folded light curve with the best-fitting transit model overlaid as a red line.

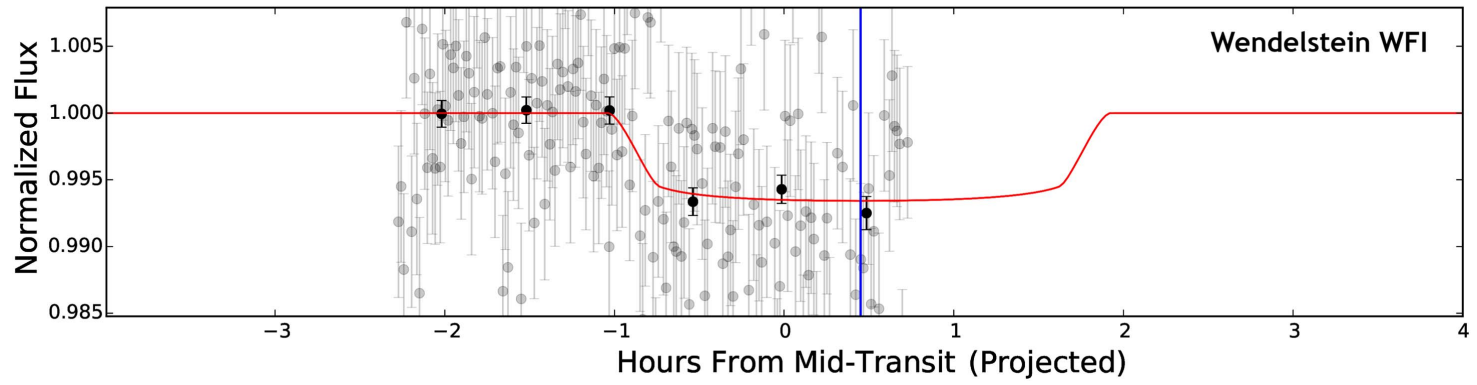

Figure 2. Normalized photometry in the $i^{\prime}$-band for K2-95, recorded with the Wendelstein WFI. We overlaid the best-fitting transit model from the $K 2$ data, adapted with appropriate quadratic limb darkening parameters for the $i^{\prime}$-band. The binned points (black) agree very well with the model (red line); however, the transit was shifted by about 27 minutes (new center indicated by the blue line) which indicates an error in the initial period estimate within the fitting uncertainties. The original points (light gray) are shown in the background.

$1^{\prime \prime}$ and $3^{\prime \prime}$ and cirrus activity which led to aborting the observations after about three hours, or around mid-transit. However, due to the relative isolation of the target and reference stars on the $\mathrm{CCD}$, the data were still salvageable and we could identify the transit after binning the data in 30 minutes intervals. The light curve seen in Figure 2 shows the expected transit depth of $0.7 \%$ and agrees very well with the overlaid best-fitting transit model from the $K 2$ data, adjusted for the respective $i^{\prime}$-band limb darkening coefficients. This light curve is already time-corrected and indicates a slight shift in phase. This implies that our initial period estimate may have been off by a few seconds per cycle, an effect seen in the follow-up of previous $K 2$ planet discoveries (see Beichman et al. 2016), but it is still inside the period uncertainty (see also Section 4.2) of $\approx 60 \mathrm{~s}$. Following up transiting planets over larger baselines and therefore improving period accuracy is a valuable step in preserving the ephemeris for future studies.

\subsection{IRTF/SpeX}

We observed our target with the near-infrared crossdispersed spectrograph (SpeX, Rayner et al. 2003) on the 3.0 m NASA Infrared Telescope Facility on Mauna Kea. While $K 2$ targets are already pre-characterized with broadband photometry, spectral typing is essential for more accurate stellar properties. K2-95 was observed on UT 2015 December 09 under excellent conditions with a clear sky and an average seeing of 0.15 . We used the instrument's short cross-dispersed mode (SXD) with the $0.3 \times 15^{\prime \prime}$ slit which provides a wavelength range of $0.68-2.5 \mu \mathrm{m}$ and a resolution of $R \approx$
2000. The target was placed at two locations along the slit and was observed in an ABBA pattern with $16 \times 185 \mathrm{~s}$ integrations for a total integration time of $2960 \mathrm{~s}$. For telluric correction and wavelength calibration, we observed an A0 standard star plus arc and flat lamp exposures right after the target. We reduced the data with the SpeXTool package (Vacca et al. 2003; Cushing et al. 2004) which performs flat fielding, sky subtraction, bad pixel removal and subsequently spectral extraction and combination, telluric correction, wavelength + flux calibration and order merging. We achieved a median $\mathrm{S} / \mathrm{N}$ of 70 per resolution element in the $J-(1.25 \mu \mathrm{m}), 80$ in the $H$ - $(1.6 \mu \mathrm{m})$ and 60 in the $K$-band $(2.2 \mu \mathrm{m})$. We compare the $J H K$-band spectra to late-type standards from the IRTF Spectral Library (Rayner et al. 2009), seen in Figure 3. The best visual match for K2-95 lies between M2 and M3 standards across all infrared bands.

\subsection{Keck/HIRES}

We obtained a high-resolution optical spectrum of K2-95 using the HIRES echelle spectrometer on the $10 \mathrm{~m}$ Keck I telescope (Vogt et al. 1994) on UT 2015 December 23. Highresolution spectroscopy can be used to rule out false-positive detection scenarios such as EBs by searching for secondary line features that are created by a possible companion star. Our observation followed the procedures of the California Planet Search (CPS, Howard et al. 2010). We used the "C2" decker, providing a spectral resolution of $R=55,000$, and subtracted the sky from the stellar spectrum. We utilized the HIRES exposure meter to automatically terminate the exposure when 


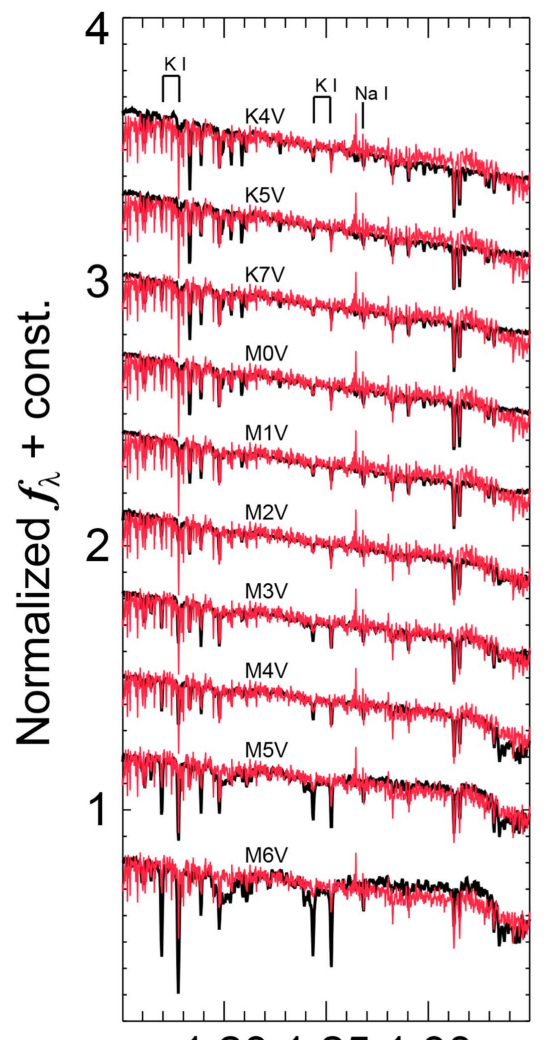

1.201 .251 .30

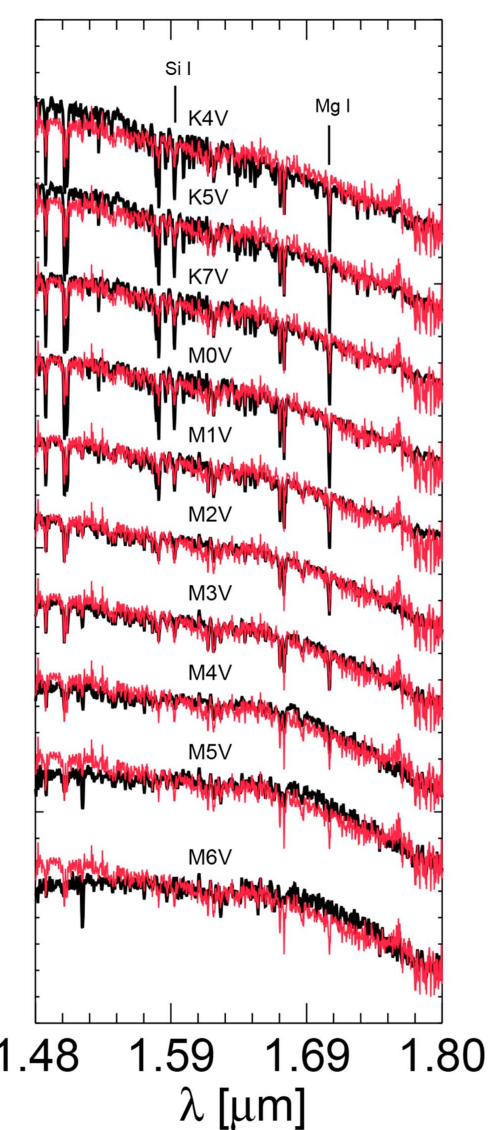

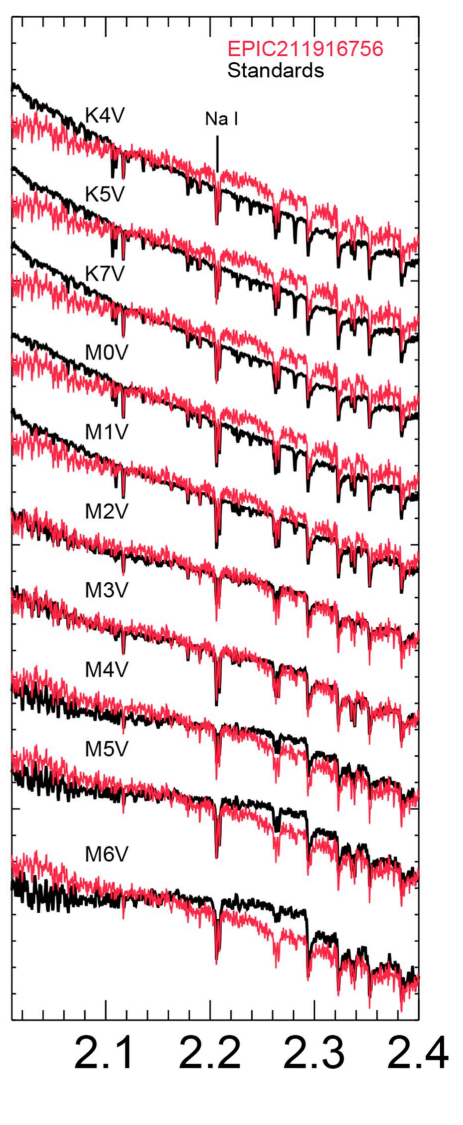

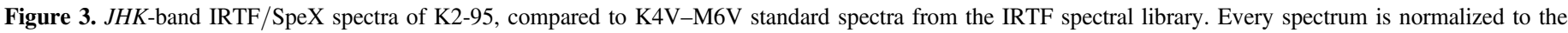

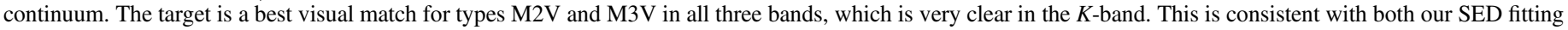
results and the spectral typing using spectroscopic indices.

$\mathrm{S} / \mathrm{N}=32$ per pixel was achieved. The HIRES spectrum was reduced using standard CPS procedures and cover $\sim 3600-8000 \AA$. Two additional spectra were obtained on UT December 24 and 29 using a redder setting of HIRES at $R=48,000$; these data are described in J. Pepper et al. (2016, in preparation).

\subsection{Keck/NIRC2}

We obtained high-resolution NIR images of K2-95 using NIRC2 on the $10 \mathrm{~m}$ Keck II telescope using the target as a natural guide star to drive the AO system. High-resolution imaging is a useful tool for constraining the probability of a blended background star. We observed the target on UT 2016 January 16 in the $K$-band, following a multi-point dither pattern with integration times short enough to avoid saturation. We used the dithered images to subtract the sky background and remove dark current, then aligned, flat-fielded, and stacked the individual images. The star appears single and has no close companions within several arcseconds. To estimate the sensitivity of the NIRC2 observations, we injected fake sources with $\mathrm{S} / \mathrm{N}=5$ into the combined image at separations that are integral multiples of the star's FWHM. We show our final image and the $5 \sigma$ sensitivity curve in the left panel of Figure 4.

\subsection{Gemini-N/DSSI}

We also obtained speckle imaging of K2-95 in two narrow band filters centered at 880 and $692 \mathrm{~nm}$ using the DSSI camera
(Horch et al. 2009) on the $8 \mathrm{~m}$ Gemini North telescope on UT 2016 January 16 . We followed a standard observing procedure where the star was centered in the field, guiding was established, and many images were taken using $60 \mathrm{~ms}$ exposures. The data were reduced and combined into a final reconstructed image using the techniques described in Horch et al. (2011) and Howell et al. (2012). These procedures perform automatic model fits (single, double, triple) and provide estimates of the magnitude difference and separation for multiple systems. K2-95 was found to be a single star. We measured the background sensitivity of the reconstructed DSSI image, using a series of concentric annuli centered on the target. The innermost annulus is at the telescope diffraction limit where our sensitivity is zero. The sensitivities in the subsequent annuli are interpolated using a cubic spline to produce a smooth sensitivity curve. The $880 \mathrm{~nm}$ reconstructed DSSI image and sensitivity curve are shown in the right panel of Figure 4.

\subsection{Archival Imaging}

Data taken from photographic plates, now digitally scanned and available online ${ }^{22}$, cover several decades of astrometry. Our target was first observed in 1954 by the Digital Sky Survey (DSS) in the red and blue channels with an additional epoch from 1989 and 1990, respectively. We show the DSS-red plates from 1954 and 1989 in Figure 5. The images

\footnotetext{
22 http://irsa.ipac.caltech.edu/applications/finderchart/
} 

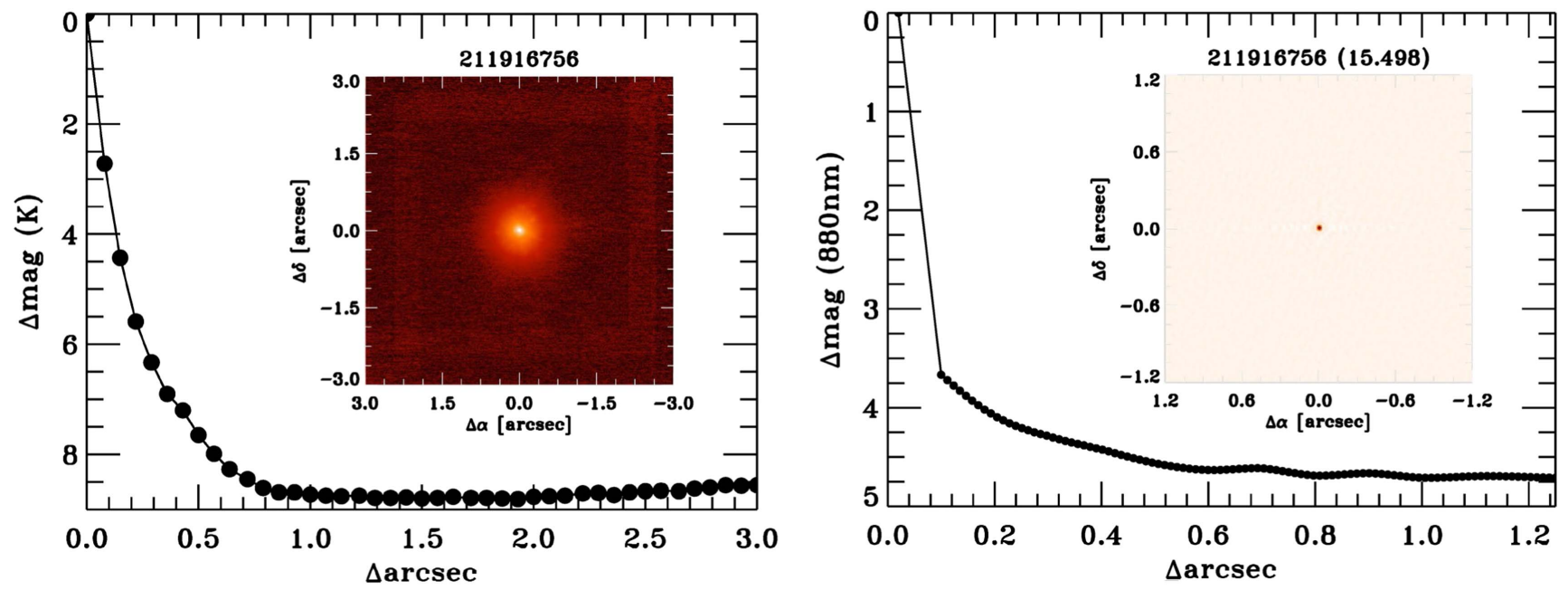

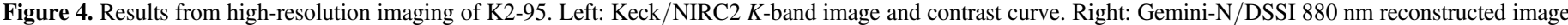

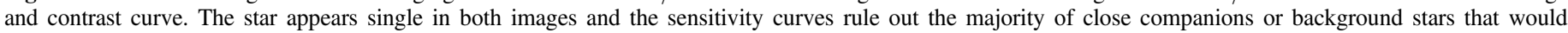
contribute significant flux to the transit light curve.

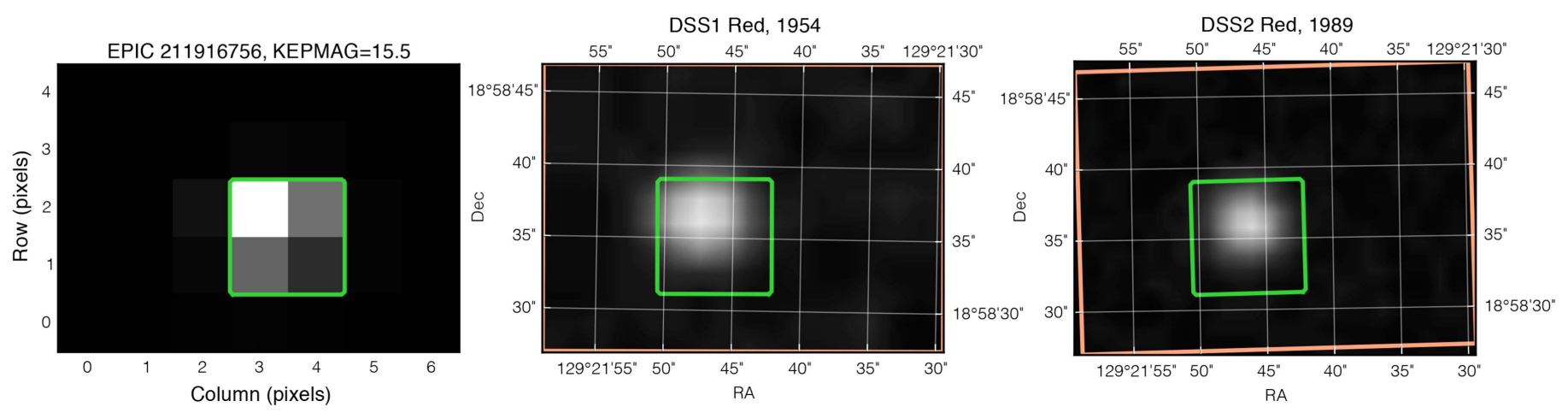

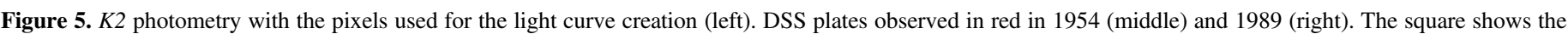

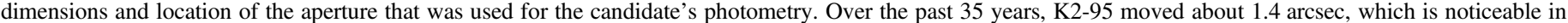
comparison of both images.

are centered on the epoch 2015 coordinates of the target in the EPIC database (08:37:27.059, +18:58:36.07) and the $K 2$ aperture is overlaid as a green square. The target's proper motion of 1.4 arcsec over the course of 35 years results in a visible shift in position, seen in comparison of the middle and left panels in Figure 5. There is no indication for a background star at the 2015 epoch position, based on the archival data. If there is a star still hidden in the background it must be quite faint, in which case it would not significantly dilute the transit signal.

\section{HOST STAR CHARACTERIZATION}

Validation of the transiting planet candidate and constraints on its physical parameters require detailed characterization of the host star's properties. We used several approaches to estimate the fundamental parameters of K2-95, including medium-resolution spectroscopy, multi-band photometry, and kinematics. We also place further constraints on close bound companions and background stars from our high-resolution spectroscopy and imaging. The results of these data are used to perform a false-positive probability (FPP) analysis of the planet candidate and estimate its properties. The final stellar properties are shown in Table 1.

\subsection{Medium-resolution Spectroscopy}

We apply the index-based methods of Mann et al. (2013a, 2013b, 2015) and equivalent width (EW)-based methods of Newton et al. $(2014,2015)$ to our SpeX spectrum in order to estimate the metallicity, temperature, radius, and luminosity of K2-95. These approaches are empirically calibrated by using wide $\mathrm{M}$ dwarf binary companions and nearby bright $\mathrm{M}$ dwarf standards with interferometrically measured radii. Our SpeX spectrum, shown in Figure 3, suffers from poor telluric correction in the $J$ - and $H$-bands. These residuals result from the long exposure time of the target which led to a large time baseline (nearly one hour) and non-ideal airmass difference $(>0.1)$ between the target and A0 calibrator. To avoid the systematic effects introduced when using the index-based methods of Mann et al. (2013b) in regions of poor telluric correction (Mann et al. 2013a; Newton et al. 2015) we use only their $K$-band relations. Prior to any analyses, the spectrum was shifted by its RV estimated via cross-correlation with an $\mathrm{M}$ dwarf standard.

To estimate the star's metallicity, we use IDL software provided by A. Mann and E. Newton. ${ }^{23}$ Using the Mann et al. (2013a) $K$-band index relations, we estimate a metallicity $[\mathrm{Fe} / \mathrm{H}]=0.09 \pm 0.09$ dex. The $K$-band EW-based methods

$\overline{23}$ https://github.com/awmann/metal, https://github.com/ernewton/nirew 
Table 1

Stellar Parameters for K2-95

\begin{tabular}{|c|c|c|}
\hline Parameter & K2-95 & Reference \\
\hline Epoch & J2000 & 1 \\
\hline R.A. & $08: 37: 27.059$ & 1 \\
\hline Decl. & $+18: 58: 36.07$ & 1 \\
\hline$\mu_{\alpha}$ & $-36.7 \pm 3.0{\text { mas } \mathrm{yr}^{-1}}^{-1}$ & 2 \\
\hline$\mu_{\delta}$ & $-15.1 \pm 3.0$ mas yr$^{-1}$ & 2 \\
\hline RV & $35.2 \pm 0.2 \mathrm{~km} \mathrm{~s}^{-1}$ & 3 \\
\hline$K_{p}$ & $15.498 \mathrm{mag}$ & 1 \\
\hline$g^{\prime}$ & $17.779 \pm 0.00240 \mathrm{mag}$ & 4 \\
\hline$r^{\prime}$ & $16.596 \pm 0.00110 \mathrm{mag}$ & 4 \\
\hline$i^{\prime}$ & $15.369 \pm 0.00079 \mathrm{mag}$ & 4 \\
\hline$z^{\prime}$ & $14.789 \pm 0.00096 \mathrm{mag}$ & 4 \\
\hline$y^{\prime}$ & $14.529 \pm 0.00220 \mathrm{mag}$ & 4 \\
\hline$J$ & $13.312 \pm 0.01700 \mathrm{mag}$ & 5 \\
\hline$H$ & $12.738 \pm 0.02300 \mathrm{mag}$ & 5 \\
\hline$K$ & $12.474 \pm 0.01900 \mathrm{mag}$ & 5 \\
\hline Spectral Type & $\mathrm{M} 3.0 \pm 0.5$ & 6 \\
\hline$T_{\text {eff }}$ & $3471 \pm 124 \mathrm{~K}$ & 6,8 \\
\hline$T_{\text {eff }}$ & $3384 \pm 100 \mathrm{~K}$ & 7 \\
\hline$d$ & $171 \pm 15 \mathrm{pc}$ & 7 \\
\hline$d$ & $172 \pm 14 \mathrm{pc}$ & 3 \\
\hline$[\mathrm{Fe} / \mathrm{H}]$ & $0.11 \pm 0.17$ & 6,8 \\
\hline Radius & $0.402 \pm 0.050 R_{\odot}$ & 6,8 \\
\hline Radius & $0.381 \pm 0.070 R_{\odot}$ & 7 \\
\hline Luminosity & $0.021 \pm 0.008 L_{\odot}$ & 6,8 \\
\hline Mass & $0.361 \pm 0.069 M_{\odot}$ & 6 \\
\hline Density & $7.81 \pm 1.90 \mathrm{~g} \mathrm{~cm}^{-3}$ & 6 \\
\hline
\end{tabular}

Note. References are: 1-EPIC Catalog, 2-Kraus \& Hillenbrand (2007), 3 this work, 4-Pan-STARRS1 3 $\pi$ catalog (version PV3), 5-2MASS catalog, 6 - this work, using (Mann et al. 2016a), 7-this work, using SED fitting from Obermeier et al. (2016), 8-this work, using Newton et al. (2015).

of Newton et al. (2014) provide $[\mathrm{Fe} / \mathrm{H}]=0.12 \pm 0.14 \mathrm{dex}$. The uncertainties were estimated using Monte Carlo sampling. These estimates are consistent with each other and also with the metallicity of Praesepe, $[\mathrm{Fe} / \mathrm{H}]=0.12 \pm 0.04 \mathrm{dex}$ (Boesgaard et al. 2013).

We estimate the effective temperature using the $K$-band index relations of Mann et al. (2013b) and the $H$-band EW-based relations of Newton et al. (2015) using IDL software provided by A. Mann and E. Newton. ${ }^{24}$ The $K$-band relations provide $T_{\text {eff }}=3460 \pm 73 \mathrm{~K}$ where the adopted uncertainty is the scatter in the polynomial relation. The $H$-band relations yield $T_{\text {eff }}=3481 \pm 100 \mathrm{~K}$. The uncertainty was estimated using Monte Carlo sampling of the measurement error in the spectrum. These consistent effective temperatures are used to estimate the radius and luminosity of the star using the aforementioned empirical calibrations. Following the Mann et al. (2013b) relations, we estimate $R_{*}=0.393 \pm 0.036 R_{\odot}$ and $L_{*}=0.017 \pm 0.006 L_{\odot}$. The Newton et al. (2015) relations provide $R_{*}=0.411 \pm 0.034 R_{\odot}$ and $L_{*}=0.024 \pm 0.006 L_{\odot}$. These fundamental parameters, estimated using different methods, are consistent at the $<1 \sigma$ level. We adopt the means of these estimates for further analyses and calculate conservative uncertainties by adding the individual errors in quadrature. The final values are provided in Table 1 . The methods of Mann et al.

\footnotetext{
$\overline{24}$ https://github.com/awmann/Teff_rad_mass_lum, https://github.com/ ernewton/nirew
}

(2013b) also provide estimates of the star's mass and density, $M_{*}=0.361 \pm 0.069 M_{\odot} \quad$ and $\rho_{*}=7.81 \pm 1.90 \mathrm{~g} \mathrm{~cm}^{-3}$, respectively. We further use the H20_K2 index (Rojas-Ayala et al. 2012) to estimate the spectral type of the star. We find K295's type to be M3.0 \pm 0.5 , consistent with visual comparisons to standard stars and our spectroscopic temperature estimates.

\subsection{SED Fitting}

We utilize the spectral energy distribution (SED) fitting code from Obermeier et al. (2016) as an additional layer of our stellar type characterization. In contrast to spectroscopy, this approach relies on broadband photometry. We extract the PanSTARRS1 $3 \pi$ data (version PV3) for this star and cross-match its coordinates with the 2MASS catalog. For the synthetic stellar SED catalog, we use the newest version of the PARSEC isochrones package (Bressan et al. 2012) which includes improvements for low-mass stars that were calibrated for Praesepe (Chen et al. 2014). The age of the cluster is known (Brandt \& Huang 2015a), therefore we restrict the synthetic model population to $800 \mathrm{Myr}$ and Praesepe's metallicity of $([\mathrm{Fe} / \mathrm{H}]=0.12 \mathrm{dex})$. Since the isochrone models are for nonrotating stars, we furthermore include a second set of isochrones at $650 \mathrm{Myr}$. We create a tenth-order polynomial to interpolate between the distance-dependent extinction values given in the 3D dust map from Green et al. (2015) and ${ }^{25}$ iteratively fit distance and extinction until both converge. We find that the final photometric fits for temperature and radius, $T_{\text {eff }}=3386 \pm 100 \mathrm{~K}$ and $R_{*}=0.43 \pm 0.070 R_{\odot}$, agree very well with the spectroscopic results and the extinction is negligible with $E(B-V)=0.0016$. The better fit was for the $650 \mathrm{Myr}$ model with a marginally better $\chi^{2}$ of 7.83 against 7.97. We also estimate a distance of $171 \pm 15 \mathrm{pc}$ which is consistent with a Praesepe cluster membership and the derived distance of $172 \pm 14 \mathrm{pc}$ based on kinematic distance and $K$-band magnitude.

\subsection{High-resolution Spectroscopy}

We use the methodology and algorithm of Kolbl et al. (2015) to search for blended background stars or close spectroscopic binary companions in our HIRES spectrum. The secondary line analysis compares the observed spectrum to a suite of about 600 well characterized, slowly rotating HIRES spectra of FGKM stars from the CPS and attempts to identify residuals consistent with a fainter secondary star. For faint, late-type stars like K2-95, this method is sensitive to spectroscopic companions projected within one half the HIRES slit width (0." 4), with approximate $V$-band fluxes as small as $3 \%$ of the primary flux and $\Delta \mathrm{RV}>10 \mathrm{~km} \mathrm{~s}^{-1}$. This sensitivity range complements our high-resolution imaging. The algorithm also measures the barycentric corrected primary RV using telluric lines. The analysis revealed no secondary lines within the above sensitivity limits. Using the color-temperature conversions of Pecaut \& Mamajek (2013), we estimate that the Kolbl et al. (2015) analysis of our HIRES spectrum rules out a large range of close companions on circular orbits down to $\sim \mathrm{M} 5.5$ types on $\sim 75$ day or shorter orbits. Additionally, we measure $\mathrm{RV}=35.2 \pm 0.2 \mathrm{~km} \mathrm{~s}^{-1}$, consistent with other Praesepe members. The combined RV constraints from our multi-epoch HIRES observations are described further in Section 4.1.

\footnotetext{
${ }^{25}$ http://argonaut.rc.fas.harvard.edu/
} 


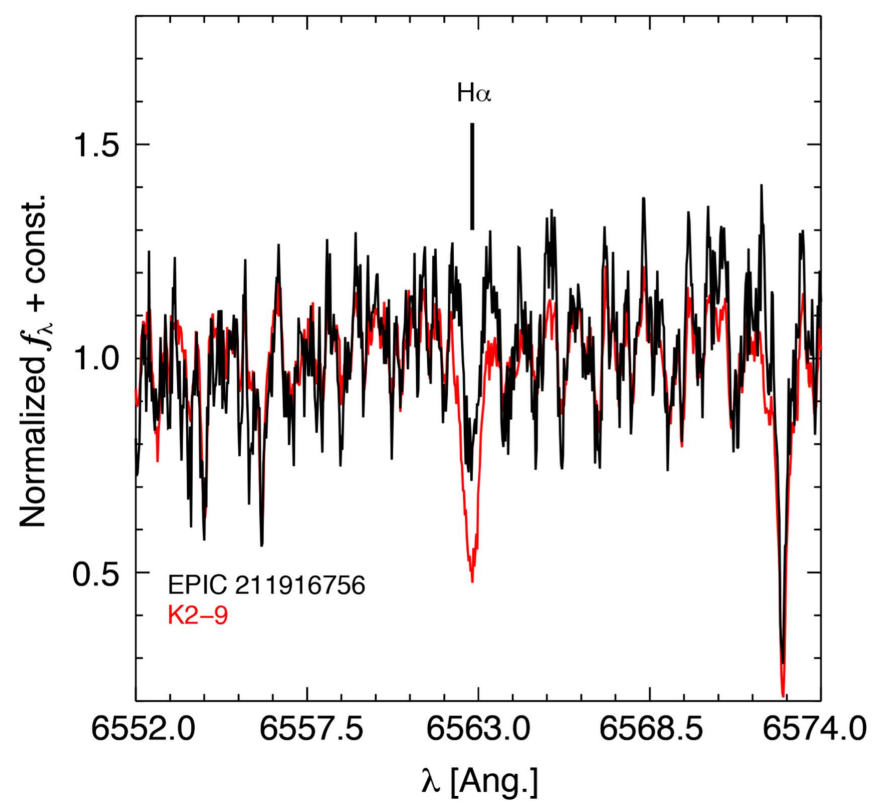

Figure 6. HIRES spectrum of K2-95 (black) centered on the $\mathrm{H} \alpha$ line compared to the known M dwarf planet host K2-9 (red). The weak activity is consistent with the lower end of the the distribution for similar spectral type stars in Praesepe and the star's slow rotation.

We also use the HIRES spectrum to investigate $\mathrm{H} \alpha$ emission at $6563 \AA$. $\mathrm{H} \alpha$ emission is a magnetic activity indicator in lowmass stars and can be used to place coarse constraints on a star's age (West et al. 2008). Kafka \& Honeycutt (2006) and Douglas et al. (2014) present $\mathrm{H} \alpha$ measurements for low-mass Praesepe members, including K2-95. They find that M3 type stars in Praesepe exhibit a wide range of emission levels, with equivalent widths (EWs) spanning approximately 0 to $-8 \AA$ (where negative EWs represent emission). K2-95 is on the low end of the emission distribution for stars of similar spectral type in their studies, with only a hint of weak emission. We show a portion of our HIRES spectrum surrounding $\mathrm{H} \alpha$ in Figure 6 compared to a field age planet host with similar spectral type, K2-9 (Montet et al. 2015; Schlieder et al. 2016). The $\mathrm{H} \alpha$ line morphology of K2-95 is different from the weak absorption observed in the older star K2-9; it exhibits narrow emission peaks in the line wings. This profile is consistent with model predictions for weakly active low-mass dwarfs (Cram \& Mullan 1979) and similar to $\mathrm{H} \alpha$ profiles observed for the slowest rotating $\mathrm{M}$ dwarfs in the younger Pleiades cluster ( $P \sim 15$ days, Stauffer et al. 2016). We conservatively estimate $\mathrm{EW}_{\mathrm{H} \alpha}=-0.1 \pm 0.1$ which is consistent with previous EW measurements and broadly consistent with expectations for an M3 dwarf in Praesepe.

We further cross-correlated our HIRES spectrum with a slowly rotating, rotationally broadened $\mathrm{M}$ dwarf standard to place constraints on the projected rotational velocity $v \sin i$. This analysis revealed that the star has a low rotational velocity with the best-match broadened spectrum having $v \sin i$ $<3 \mathrm{~km} \mathrm{~s}^{-1}$. This low $v \sin i$ and the long rotation period ( 24 days) estimated from de-trended $K 2$ photometry are consistent with the slowest rotating Praesepe $M$ dwarfs presented in Douglas et al. (2014). Both indications of slow rotation are also consistent with the low level of magnetic activity inferred from the $\mathrm{H} \alpha$ line. The slow rotation of this intermediate-age $\mathrm{M}$ dwarf is remarkable when considering its close-in planet (see
Section 4) and may indicate differences in angular momentum evolution due to initial conditions, the primordial disk, planet formation, or planet migration. In contrast, the very similar Hyades M dwarf planetary system K2-25 is among the fastest rotating $\mathrm{M}$ dwarfs in that cluster with a period of $\sim 1.9$ days (Douglas et al. 2014; David et al. 2016a; Mann et al. 2016a).

\subsection{High-resolution Imaging}

Using the Gemini/DSSI speckle results, we can constrain the contamination from nearby sources. The DSSI data in the $880 \mathrm{~nm}$ band provide the best constraints to bound and background companions at very close separations. At a separation of 0 " 1 , our sensitivity to companions is $\Delta \operatorname{mag}(880 \mathrm{~nm}) \approx 3.5 \mathrm{mag}$.

Our Keck/NIRC2 AO imaging provides deeper constraints on close background and bound companions at larger separations. At separations of $0 . ! 2$ and 0 !! 5 , we estimate sensitivity to companions with $\Delta K \approx 5 \mathrm{mag}$ and $\Delta K \approx 8 \mathrm{mag}$, respectively. This effectively rules out all background sources within these separations that could contribute significant flux to the light curve. We use the relations of Pecaut \& Mamajek (2013) to estimate that our combined Keck and Gemini imaging rule out all bound companions at the same distance down to the hydrogen burning limit at separations of 0 ". 1 (17 au) and well into the brown dwarf regime at $\gtrsim 0$ " 5 (86 au). We use both our Keck/NIRC2 and Gemini/DSSI contrast curves as constraints in the FPP analysis.

\subsection{Cluster Membership, Kinematics, and Age}

K2-95 was first identified as a candidate member of Praesepe by Williams et al. (1994) and was subsequently included in the proposed member lists of several works including Hambly et al. (1995) and Adams et al. (2002). Kraus \& Hillenbrand (2007) combined photometry, astrometry, and the kinematics of well defined cluster members in a maximum likelihood analysis to estimate that K2-95 has a $>99 \%$ probability of cluster membership. To further investigate its Praesepe membership, we use the star's partial kinematics and the methods described in Lépine \& Simon (2009) to estimate a kinematic distance $\left(d_{\text {kin }}\right)$ and predicted radial velocity $\left(\mathrm{RV}_{p}\right)$. In the analysis we adopt the UVW Galactic velocities of Praesepe from van Leeuwen (2009) and estimate errors using Monte Carlo sampling. We find $d_{\text {kin }}=172 \pm 14 \mathrm{pc}$, consistent with our SED-based estimate of the star's distance and the average cluster distance, and $\mathrm{RV}_{\mathrm{p}}=34.1 \pm 0.9 \mathrm{~km} \mathrm{~s}^{-1}$, consistent with our measured RV from Keck/HIRES spectroscopy. The consistency of these predictions and measurements, along with the spectroscopic indications of activity in our HIRES data, confirm the membership of K2-95 in the low-mass population of Praesepe which places a conservative constraint on its age of 600-800 Myr. We also use the kinematic distance and $K$-band magnitude of the star to determine its luminosity using the conversions of Pecaut \& Mamajek (2013). We estimate $L_{*}=0.021 \pm 0.003 L_{\odot}$. At the age of Praesepe, an M3 dwarf is expected to be on the main sequence and has stopped radial contraction. We can therefore combine our measured effective temperature and luminosity through the Stefan-Boltzmann law to estimate the star's radius, $R_{*}=0.40 \pm 0.01 R_{\odot}$. These alternative estimates of the star's fundamental parameters are consistent with those from our SpeX spectroscopy and SED fitting. 


\section{PLANET VALIDATION}

\subsection{False Positive Probability}

For a transiting planet-signal, there are five common sources of false-positive identification or transit mischaracterization, most of which are created by EBs:

1. Background star.

2. Blended EB system.

3. Unblended EB system.

4. Double-period EB system.

5. Hierarchical EB companion.

Our collected data in form of photometry, spectroscopy, and high-resolution imaging can be used to place a number of constraints on the data to limit or even completely rule out all of the above scenarios. In the $K 2$ data, we detected no secondary eclipse that would be indicative of an EB. Based on archival and high-resolution imaging and high-resolution spectra, a background source is strongly constrained to less than $3 \%$ of flux dilution and can be ruled out completely for a separation of more than 0.2 arcsec. This makes any kind of background blend or triple system highly improbable. In a case where it did exist, it would not impact the planet parameters significantly.

For a more quantitative assessment, we utilize the FPP calculator vespa (Morton 2012, 2015) which is open source and freely available online. ${ }^{26}$ This program compares the light curve to transit shapes created by false-positive sources and combines this with priors about stellar population, multiplicity frequencies and the planet occurrence rate for the corresponding fitted parameters. We supply the algorithm with all of our determined constraints, including stellar photometry from 2MASS and WISE, contrast curves from high-angular resolution imaging and the light curve from $K 2$. Furthermore, we also extract the photometric light curve from Vanderburg \& Johnson (2014), remove the periodic modulations, recover the signal with the Pan-Planets signal detection pipeline (Obermeier et al. 2016) and then perform the same analysis. This way, we end up with an independent confirmation based on a different data reduction and signal detection routine. Based on all of the above constraints, the results from vespa rule out all false-positive scenarios to a FPP of less than $0.02 \%$ for both analyses. While vespa does not fit blended planetary systems, there are strong constraints on this scenario based on high-resolution imaging and the upper limit of $3 \%$ in flux dilution for background sources which makes this scenario highly unlikely. As an additional layer of security, we furthermore obtained three $\mathrm{RV}$ points based on high-resolution spectroscopy in order to constrain any EB or double-period EB scenario.

\subsubsection{Unblended EB System}

The unblended EB scenario consists of very shallow eclipses of both stars which may emulate a planet's transit light curve. There are many constraints to this scenario in the case of K2-95: the signal of a secondary eclipse is absent in the light curve data and the high-resolution spectroscopy excludes the presence of a second star down to $10 \mathrm{~km} \mathrm{~s}^{-1}$ and $3 \%$ flux. Based on both our own observation with HIRES and the two additional data points from J. Pepper et al. (2016, in

\footnotetext{
$\overline{26}$ https://github.com/timothydmorton/vespa
}
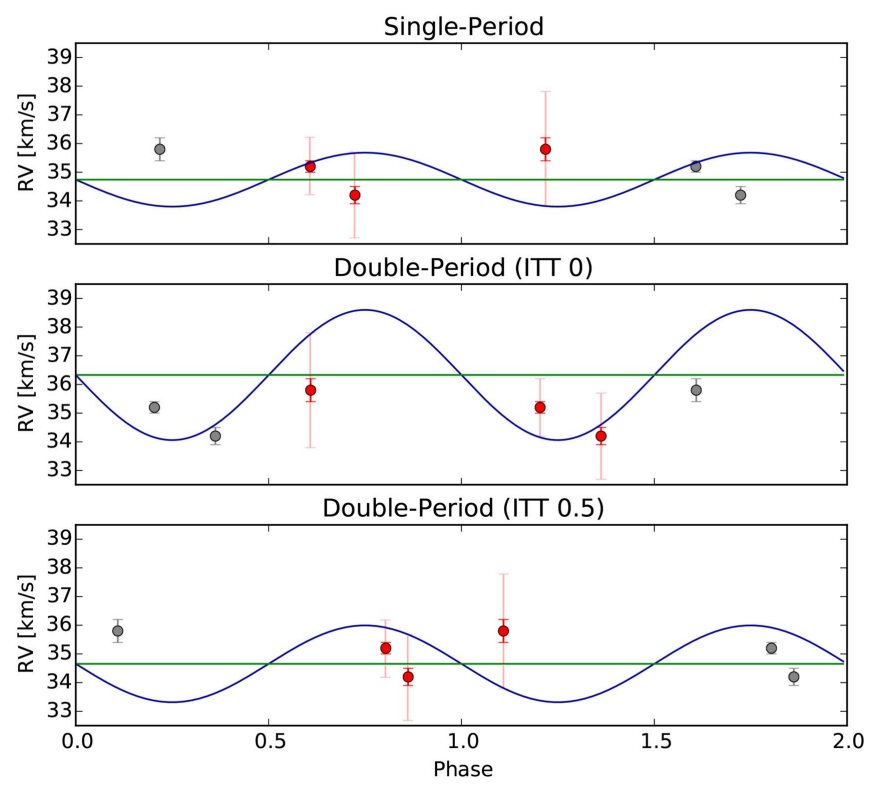

Figure 7. Radial velocity for K2-95 in the single-period (top) and doubleperiod scenario (middle+bottom), phased to the corresponding period and ITT scenario. ITT stands for the initial time phase of the first recorded eclipse, i.e., whether the primary or secondary star eclipsed first. The RV curve (blue) shows the maximum amplitude consistent with the points at $5 \sigma$. Two phases are shown for better clarity with repeated points grayed out and the error bars of the points are given in $1 \sigma$ (red) and $5 \sigma$ (light red). The green line shows the baseline fit.

preparation), we cover a time baseline of six days that we use to construct a $5 \sigma$ upper limit for the maximum RV amplitude that could still fit to the data and is shown in Figure 7 in the top panel. The result is an amplitude $941 \mathrm{~m} \mathrm{~s}^{-1}$ which equates to $5.25 M_{\mathrm{J}}$, a giant planet. These limitations mean that this signal cannot be modeled as an unblended EB system.

\subsubsection{Double-period EB System}

The double-period case is different to other scenarios in that it assumes an EB system in which both partners have the same size and eclipse each other. This changes fundamental parameters such as the relative eclipse duration and impacts limits for the secondary eclipse; strong constraints make this scenario more likely.

Both partners must have similar radii in this case. As in the single-period EB scenario, we combine our HIRES RV measurement with the two measurements presented in J. Pepper et al. (2016, in preparation). We again set limits for which the RV curve is outside of $5 \sigma$ of the individual points, which is shown in Figure 7 in the middle and bottom panels. Two cases have to be considered, depending on whether the initial transit time (ITT) was at phase 0 or 0.5 (ITT 0 and ITT 0.5 , respectively). The subsequent limit is an RV of $2270 \mathrm{~m} \mathrm{~s}^{-1}$ for ITT 0 and $1343 \mathrm{~m} \mathrm{~s}^{-1}$ for ITT 0.5 . Taking the stellar mass determined by medium-resolution spectroscopy and assuming a circular orbit, this translates to $15.46 M_{\mathrm{J}}$, a low-mass brown dwarf, or $9.14 M_{\mathrm{J}}$, a giant planet. Any stellar companion would produce a much stronger RV signal and an eclipse of the primary in front of a brown dwarf cannot create such a strong signal.

Additionally, K2-95 has a probability of more than $99 \%$ for being a member of the Praesepe cluster, which means that the baseline of the fitted RV curve for the case of ITT 0 , 
Table 2

Best-fitting Properties of K2-95 and Its Planet Based on the BATMAN Code

\begin{tabular}{lcl}
\hline \hline Parameter & Units & $\mathrm{K} 2-95$ \\
\hline$T_{0}$ & $\mathrm{BJD}_{\mathrm{TDB}}-2454833$ & $2338.1477_{-0.0019}^{+0.0018}$ \\
$P$ & day & $10.13389_{-0.00077}^{+0.00068}$ \\
$i$ & $\mathrm{deg}$ & $88.77_{-1.59}^{+0.86}$ \\
$R_{P} / R_{\star}$ & $\%$ & $7.86_{-0.93}^{+1.69}$ \\
$R_{\star} / a$ & $\ldots$ & $0.0400_{-0.0068}^{+0.0187}$ \\
$T_{14}$ & $\mathrm{hr}$ & $2.84_{-0.26}^{+0.36}$ \\
$T_{23}$ & $\mathrm{hr}$ & $2.18_{-0.72}^{+0.26}$ \\
$a$ & $\mathrm{au}$ & $0.0653_{-0.00045}^{+0.0039}$ \\
$R_{P}$ & $R_{E}$ & $3.47_{-0.53}^{+0.78}$ \\
$R_{\star}$ & $R_{\odot}$ & $0.402_{-0.050}^{+0.050}$ \\
$M_{\star}$ & $M_{\odot}$ & $0.361_{-0.069}^{+0.069}$ \\
\hline
\end{tabular}

$\mathrm{RV}=36.3 \mathrm{~km} \mathrm{~s}^{-1}$, should be consistent with the cluster $\mathrm{RV}_{\mathrm{p}}=34.1 \pm 0.9 \mathrm{~km} \mathrm{~s}^{-1}$. ITT 0 is only consistent at $3 \sigma$ which further decreases the likelihood of this scenario. In contrast, the RV baseline for a single-period transiting planet scenario is very consistent with a best fit of $34.8 \mathrm{~km} \mathrm{~s}^{-1}$.

Therefore, in combination with all of the other constraints (e.g., AO imaging, archival optics, stellar characterization), the transit signal cannot be modeled successfully with this scenario and we can rule it out.

\subsection{Planet Parameters}

We analyze the light curve of K2-95 with a approach similar to the one described in more detail by Crossfield et al. (2015). ${ }^{27}$ In brief: relying on the emcee package (Foreman-Mackey et al. 2013), we use the open-source BATMAN light curve code (Kreidberg 2015) which we optimize for long-cadence data. Utilizing the free and open-source LDTk/pyLDTk package from Parviainen \& Aigrain $(2015)^{28}$, we propagate our measured $T_{\text {eff, }}$ surface gravity, metallicity and their respective uncertainties into limb-darkening coefficients for use as priors in our fit. The overall fitted parameters in our analysis are the candidate's orbital period $\mathrm{P}$, initial transit time $T_{0}$, inclination $i$, eccentricity $e$, longitude $\omega$, scaled semimajor axis $a / R_{\star}$ and the fractional candidate radius $R_{p} / R_{\star}$. The starting parameters for the fit are taken from our TERRA output. In the fit, we assume a linear ephemeris for the transits which should be a valid simplification since there is no evidence for any kind of TTV in the light curve. The bestfitting properties and their uncertainties are shown in Table 2. We estimate the planet's mass using the mass-radius relation ${ }^{29}$ provided by Wolfgang \& Lopez (2015) and Wolfgang et al. (2015), $M / M_{\oplus}=2.7\left(R / R_{\oplus}\right)^{1.3}$ to $M_{\mathrm{P}}=13.71 \pm 3.62 M_{\oplus}{ }^{30}$ However, using the relation provided by Weiss \& Marcy (2014), $M / M_{\oplus}=2.69\left(R / R_{\oplus}\right)^{0.93}$, we get $M_{\mathrm{P}}=8.77_{-0.53}^{+1.88} M_{\oplus}$. A third mass-radius relation, published by Chen \& Kipping

\footnotetext{
${ }^{27}$ Further information about the most up-to-date method will be found in Crossfield et al. (2016).

28 https://github.com/hpparvi/ldtk

29 And their code: https://github.com/dawolfgang/MRrelation.

30 The code cannot handle asymmetrical errors, hence we selected the larger of both uncertainties.

31 https://github.com/chenjj2/forecaster

32 The code cannot handle asymmetrical errors, hence we used the larger of both uncertainties.
}

$(2016)^{31,32}$, yields $M_{\mathrm{P}}=8.26_{-0.50}^{+1.77} M_{\oplus}$ based on the relation $M / M_{\oplus}=\left(R / R_{\oplus}\right)^{1.70}$. The mass-radius models lead to different estimates of the planet's mass. While the results from Wolfgang \& Lopez (2015) are higher than the other two, the difference is still small enough for the masses to be marginally consistent with each other. The absence of TTVs in the system means that the mass cannot be determined through other means as of now. We estimate the RV amplitude of this planet to be $6.8 \pm 1.8 \mathrm{~m} \mathrm{~s}^{-1}$, based on the Wolfgang \& Lopez (2015) results.

\section{DISCUSSION}

So far, only very few planets have been detected in clusters, even fewer with the transit method. K2-95b is only the third known planet in an open cluster that orbits around an M dwarf. Assuming a typical density of small gas planets, it probably belongs to the class of Neptune-size planets with a similar chemical composition and $\mathrm{H} / \mathrm{He}$ atmospheres (Marcy et al. 2014; Weiss \& Marcy 2014; Rogers 2015).

However, it is remarkable that the occurrence rate of planets with the radius and period of both K2-25 (David et al. 2016a; Mann et al. 2016a) and K2-95b is very low around field stars (Dressing \& Charbonneau 2015; Mulders et al. 2015). Furthermore, the recently discovered planet K2-33b in the open cluster Upper Scorpius (David et al. 2016b; Mann et al. 2016b) exhibits an unusually large radius as well. While there are four discovered systems with planet radii higher than $\mathrm{K} 2-95 \mathrm{~b}$ and K2-25b, those planets are even larger and orbit higher-mass stars. Furthermore, their received stellar flux appears to be significantly higher. The distribution of planetary radii and received radiation against the host star mass are shown in Figure 8. We placed the following restrictions: all planets in this figure have to be confirmed and we extract the most recent planetary and stellar parameters from the NASA exoplanet archive (Akeson et al. 2013). Furthermore, the host star radii have to be below $0.5 R_{\odot}$ and the planet irradiance is calculated when missing.

The probability of detecting two such planets in a cluster without any detections in the larger field star sample, plus another detection in a scarcely populated region of larger-radius planets, is too low for this detection to be random chance. We present three possible implications from this.

1. The formation of short-period planets is different in clusters due to gravitational interactions during migration. An indication for this may be the higher occurrence rate of hot Jupiters in M67 measured by Brucalassi et al. (2016). However, Meibom et al. (2013) found an occurrence rate similar to that of field stars for NGC 6811. As of now, there is insufficient information to confirm this theory.

2. M dwarfs remain active for several hundred Myr after their formation to a varying degree (Shkolnik \& Barman 2014). Strong UV emission in the relatively young Hyades and Praesepe cluster $M$ dwarfs might lead to the inflation seen in Figure 8. However, no emission could be detected by GALEX down to 19.9 mag in the far UV and $20.8 \mathrm{mag}$ in the NUV (Bianchi et al. 2011). Young planets may also be larger due to initial heat from formation (Mann et al. 2016a).

3. It is possible that this is due to a selection bias since young stars are more active. Their variability may mask 


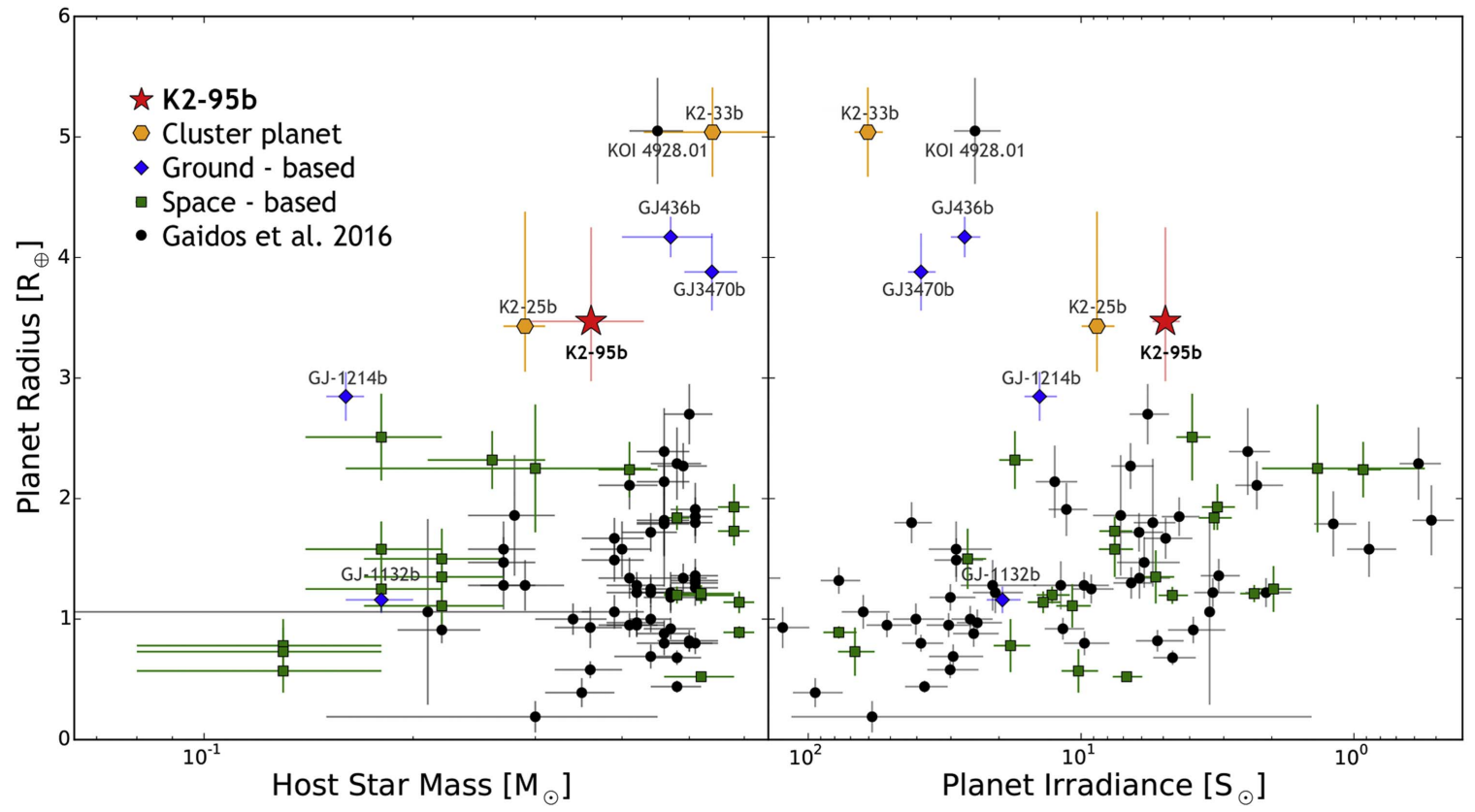

Figure 8. Planet radius as a function of the host star mass (left) and received radiation (right), comparing our discovery K2-95 (red star) to planet detections in open clusters (orange hexagons), ground-based surveys (blue diamonds), space-based (Kepler $+K 2$ ) surveys (green squares), and revised values for several Kepler planets from Gaidos et al. (2016) (black circles). Similar to Mann et al. (2016a), only stellar radii below $0.5 R_{\odot}$ and periods below 100 days were included. Two exceptions to those criteria are RV-planet GJ 3470b and K2-33b which were added due to their similarity despite a larger host star radius. All RV detections and inflated planets are labelled.

many of the small-planet transit signals, leading to a perceived imbalance. However, K2-95 is only weakly active so while a selection bias may exist, it is unlikely to be the sole reason.

Measuring the stellar UV activity and the planet's mass will allow us to determine whether the reason behind the large radii is inflation due to strong UV irradiation and/or initial heat. If that were the case, they could be seen as outliers of the general planet mass-radius relation and might be similar to GJ 436b, a Neptune-sized planet first detected by RV measurements (Butler et al. 2004) that is showing visible transits (Gillon et al. 2007) and appears to evaporate (Ehrenreich et al. 2015). However, as can be seen in Figure 8 on the right, GJ 436b receives several times of K2-95b's radiation so it is questionable whether this may apply here. Both cluster detections also orbit noticeably smaller stars than the larger Neptunian planets.

Besides this anomaly, K2-95b is also intriguing for a number of other reasons, especially for having a well-determined distance, (young) age, and metallicity. Only very few planets are known around relatively young stars and new detections will contribute toward establishing a more accurate timeline of planetary development.

Assuming a circular orbit-considering the transit duration shows no indication of ellipticity this is a valid simplificationand using the mass-radius relation from Wolfgang \& Lopez (2015), we calculate the RV amplitude to be $6.8 \pm 1.8 \mathrm{~m} \mathrm{~s}^{-1}$. While an accuracy of $1 \mathrm{~m} \mathrm{~s}^{-1}$ is entirely feasible today with instruments like HIRES or HARPS, the target is too faint to realistically achieve this with today's telescopes in reasonable observing times. However, future dedicated infrared spectrographs such as IRD and HPF (Kotani et al. 2014 and Mahadevan et al. 2012, respectively) will allow the determination of the planet's mass. This in turn will provide additional data for the calibration of the mass-radius relation of Neptune- sized gas planets. Next-generation large telescopes such as the E-ELT or the TMT may enable a detailed study of the planet's atmosphere.

As an alternative to spectroscopy, multi-band photometry enables a more detailed study of the planet, even for stars that are too faint for atmosphere spectroscopy. Depending on the photometric band, the transit eclipse depth may vary due to Rayleigh scattering or varying opacities which allows us to model the atmosphere (Mislis et al. 2010; Southworth et al. 2012; Mancini et al. 2013; Ciceri et al. 2016). While this is possible to do with single-band photometric instruments, simultaneous multi-band capture with GROND (Greiner et al. 2008) or the upcoming three-channel imager $3 \mathrm{KK}$ at Mt. Wendelstein (Lang-Bardl et al. 2010) would be much more advantageous.

\section{SUMMARY}

We report on the discovery of a Neptune-sized planet in the Beehive cluster (Praesepe) that orbits a cool dwarf star. Discussing and subsequently ruling out each possible falsepositive detection scenario, we validate the planetary nature of this candidate. Using detailed follow-up, including groundbased transit recording, spectroscopy and high-resolution imaging, we characterize both the host star and its planet. We noticed a radius anomaly for this planet and the previously detected K2-25b, both planets around M dwarfs in clusters. Both of them possess radii that are in a region seemingly unpopulated by planets orbiting comparable field stars. Detailed study and future observations will reveal whether this is due to different planet formation or evolution in open clusters.

We thank the staff of the Wendelstein observatory for technical help and strong support during the data acquisition, 
including observing the target for us. We especially thank Ulrich Hopp for his constructive input during and after observations.

The research of J.E.S. was supported by an appointment to the NASA Postdoctoral Program at NASA Ames Research Center, administered by Universities Space Research Association through a contract with NASA.

E.A.P. acknowledges support through a Hubble Fellowship.

Some of the data presented herein were obtained at the W.M. Keck Observatory (which is operated as a scientific partnership among Caltech, UC, and NASA) and at the Infrared Telescope Facility (IRTF, operated by UH under NASA contract NNH14CK55B). The authors wish to recognize and acknowledge the very significant cultural role and reverence that the summit of Mauna Kea has always had within the indigenous Hawaiian community. We are most fortunate to have the opportunity to conduct observations from this mountain.

Based on observations obtained at the Gemini Observatory, which is operated by the Association of Universities for Research in Astronomy, Inc., under a cooperative agreement with the NSF on behalf of the Gemini partnership: the National Science Foundation (United States), the National Research Council (Canada), CONICYT (Chile), Ministerio de Ciencia, Tecnología e Innovación Productiva (Argentina), and Ministério da Ciência, Tecnologia e Inovação (Brazil).

Facilities: Kepler, K2, IRTF (SpeX), Keck: I (HIRES), Keck: II (NIRC2), Gemini-N (DSSI).

Software: vespa (Morton 2012, 2015), emcee (ForemanMackey et al. 2013), BATMAN (Kreidberg 2015), LDTk/ pyLDTk (Parviainen \& Aigrain 2015).

\section{REFERENCES}

Adams, F. C., Proszkow, E. M., Fatuzzo, M., \& Myers, P. C. 2006, ApJ, 641, 504

Adams, J. D., Stauffer, J. R., Skrutskie, M. F., et al. 2002, AJ, 124, 1570

Akeson, R. L., Chen, X., Ciardi, D., et al. 2013, PASP, 125, 989

Allard, F., Homeier, D., \& Freytag, B. 2012, RSPTA, 370, 2765

Baraffe, I., Homeier, D., Allard, F., \& Chabrier, G. 2015, A\&A, 577, A42

Bianchi, L., Herald, J., Efremova, B., et al. 2011, Ap\&SS, 335, 161

Boesgaard, A. M., Roper, B. W., \& Lum, M. G. 2013, ApJ, 775, 58

Bonnell, I. A., Smith, K. W., Davies, M. B., \& Horne, K. 2001, MNRAS, 322,859

Bramich, D. M., \& Horne, K. 2006, MNRAS, 367, 1677

Brandt, T. D., \& Huang, C. X. 2015a, ApJ, 807, 24

Brandt, T. D., \& Huang, C. X. 2015b, ApJ, 807, 58

Bressan, A., Marigo, P., Girardi, L., et al. 2012, MNRAS, 427, 127

Brucalassi, A., Pasquini, L., Saglia, R., et al. 2014, A\&A, 561, L9

Brucalassi, A., Pasquini, L., Saglia, R., et al. 2016, A\&A, 592, L1

Butler, R. P., Vogt, S. S., Marcy, G. W., et al. 2004, ApJ, 617, 580

Charbonneau, D., Brown, T. M., Latham, D. W., \& Mayor, M. 2000, ApJL, 529, L45

Chen, J., \& Kipping, D. M. 2016, arXiv:1603.08614

Chen, Y., Girardi, L., Bressan, A., et al. 2014, MNRAS, 444, 2525

Christiansen, J. L., Clarke, B. D., Burke, C. J., et al. 2015, ApJ, 810, 95

Ciceri, S., Mancini, L., Southworth, J., et al. 2016, MNRAS, 456, 990

Claret, A., Hauschildt, P. H., \& Witte, S. 2012, A\&A, 546, A14

Cram, L. E., \& Mullan, D. J. 1979, ApJ, 234, 579

Crossfield, I. J. M., Ciardi, D. R., Petigura, E. A., et al. 2016, ApJS, 226, 7

Crossfield, I. J. M., Petigura, E., Schlieder, J. E., et al. 2015, ApJ, 804, 10

Cushing, M. C., Vacca, W. D., \& Rayner, J. T. 2004, PASP, 116, 362

David, T. J., Conroy, K. E., Hillenbrand, L. A., et al. 2016a, AJ, 151, 112

David, T. J., \& Hillenbrand, L. A. 2015, ApJ, 804, 146

David, T. J., Hillenbrand, L. A., Petigura, E. A., et al. 2016b, Natur, 534, 658

Debes, J. H., \& Jackson, B. 2010, ApJ, 723, 1703

Dobbie, P. D., Napiwotzki, R., Burleigh, M. R., et al. 2006, MNRAS, 369, 383

Donati, J. F., Moutou, C., Malo, L., et al. 2016, Natur, 534, 662

Dotter, A., Chaboyer, B., Jevremović, D., et al. 2008, ApJS, 178, 89

Douglas, S. T., Agüeros, M. A., Covey, K. R., et al. 2014, ApJ, 795, 161
Dressing, C. D., \& Charbonneau, D. 2015, ApJ, 807, 45

Ehrenreich, D., Bourrier, V., Wheatley, P. J., et al. 2015, Natur, 522, 459

Espinoza, N., \& Jordán, A. 2015, MNRAS, 450, 1879

Foreman-Mackey, D., Hogg, D. W., Lang, D., \& Goodman, J. 2013, PASP, 125,306

Fossati, L., Bagnulo, S., Landstreet, J., et al. 2008, A\&A, 483, 981

Gaidos, E., Mann, A. W., Kraus, A. L., \& Ireland, M. 2016, MNRAS, 457, 2877

Gilliland, R. L., Brown, T. M., Guhathakurta, P., et al. 2000, ApJL, 545, L47

Gillon, M., Pont, F., Demory, B.-O., et al. 2007, A\&A, 472, L13

Green, G. M., Schlafly, E. F., Finkbeiner, D. P., et al. 2015, ApJ, 810, 25

Greiner, J., Bornemann, W., Clemens, C., et al. 2008, PASP, 120, 405

Haisch, K. E., Jr., Lada, E. A., \& Lada, C. J. 2001, ApJL, 553, L153

Hambly, N. C., Steele, I. A., Hawkins, M. R. S., \& Jameson, R. F. 1995 , A\&AS, 109, 29

Henry, G. W., Marcy, G. W., Butler, R. P., \& Vogt, S. S. 2000, ApJL, 529, L41

Henry, T. J., Jao, W.-C., Subasavage, J. P., et al. 2006, AJ, 132, 2360

Hopp, U., Bender, R., Grupp, F., et al. 2014, Proc. SPIE, 9145, 91452D

Horch, E. P., Gomez, S. C., Sherry, W. H., et al. 2011, AJ, 141, 45

Horch, E. P., Veillette, D. R., Baena Gallé, R., et al. 2009, AJ, 137, 5057

Howard, A. W., Johnson, J. A., Marcy, G. W., et al. 2010, ApJ, 721, 1467

Howell, S. B., Horch, E. P., Everett, M. E., \& Ciardi, D. R. 2012, PASP, 124,1124

Howell, S. B., Sobeck, C., Haas, M., et al. 2014, PASP, 126, 398

Howell, S. B., VanOutryve, C., Tonry, J. L., Everett, M. E., \& Schneider, R. 2005, PASP, 117, 1187

Kafka, S., \& Honeycutt, R. K. 2006, AJ, 132, 1517

Kolbl, R., Marcy, G. W., Isaacson, H., \& Howard, A. W. 2015, AJ, 149, 18

Kopparapu, R. K., Ramirez, R. M., SchottelKotte, J., et al. 2014, ApJL, 787, L29

Kosyra, R., Gössl, C., Hopp, U., et al. 2014, ExA, 38, 213

Kotani, T., Tamura, M., Suto, H., et al. 2014, Proc. SPIE, 9147, 914714

Kraus, A. L., \& Hillenbrand, L. A. 2007, AJ, 134, 2340

Kreidberg, L. 2015, PASP, 127, 1161

Lang-Bardl, F., Hodapp, K., Jacobson, S., et al. 2010, Proc. SPIE, 7735 , 773530

Lépine, S., \& Simon, M. 2009, AJ, 137, 3632

Lovis, C., \& Mayor, M. 2007, A\&A, 472, 657

Madsen, S., Dravins, D., \& Lindegren, L. 2002, A\&A, 381, 446

Mahadevan, S., Ramsey, L., Bender, C., et al. 2012, Proc. SPIE, 8446, 84461S

Majaess, D. J., Turner, D. G., Lane, D. J., \& Krajci, T. 2011, JAVSO, 39, 219

Malavolta, L., Nascimbeni, V., Piotto, G., et al. 2016, A\&A, 588, A118

Mancini, L., Ciceri, S., Chen, G., et al. 2013, MNRAS, 436, 2

Mandel, K., \& Agol, E. 2002, ApJL, 580, L171

Mann, A. W., Brewer, J. M., Gaidos, E., Lépine, S., \& Hilton, E. J. 2013, AJ, 145,52

Mann, A. W., Feiden, G. A., Gaidos, E., Boyajian, T., \& von Braun, K. 2015 , ApJ, 804, 64

Mann, A. W., Gaidos, E., \& Ansdell, M. 2013, ApJ, 779, 188

Mann, A. W., Gaidos, E., Mace, G. N., et al. 2016a, ApJ, 818, 46

Mann, A. W., Newton, E. R., Rizzuto, A. C., et al. 2016b, arXiv:1604.06165

Marcy, G. W., Isaacson, H., Howard, A. W., et al. 2014, ApJS, 210, 20

Meibom, S., Torres, G., Fressin, F., et al. 2013, Natur, 499, 55

Mislis, D., Schröter, S., Schmitt, J. H. M. M., Cordes, O., \& Reif, K. 2010, A\&A, 510, A107

Montet, B. T., Morton, T. D., Foreman-Mackey, D., et al. 2015, ApJ, 809, 25 Morton, T. D. 2012, ApJ, 761, 6

Morton, T. D. 2015, Astrophysics Source Code Library, VESPA: False positive probabilities calculator, ascl:1503.011

Mulders, G. D., Pascucci, I., \& Apai, D. 2015, ApJ, 814, 130

Newton, E. R., Charbonneau, D., Irwin, J., et al. 2014, AJ, 147, 20

Newton, E. R., Charbonneau, D., Irwin, J., \& Mann, A. W. 2015, ApJ, 800,85

Obermeier, C., Koppenhoefer, J., Saglia, R. P., et al. 2016, A\&A, 587, A49

Parviainen, H. 2015, Astrophysics Source Code Library, PyLDTk: Python toolkit for calculating stellar limb darkening profiles and model-specific coefficients for arbitrary filters, ascl:1510.003

Parviainen, H., \& Aigrain, S. 2015, MNRAS, 453, 3821

Pecaut, M. J., \& Mamajek, E. E. 2013, ApJS, 208, 9

Pepper, J. 2016, ApJ, submitted

Petigura, E. A., Marcy, G. W., \& Howard, A. W. 2013, ApJ, 770, 69

Petigura, E. A., Schlieder, J. E., Crossfield, I. J. M., et al. 2015, ApJ, 811, 102

Quinn, S. N., White, R. J., Latham, D. W., et al. 2012, ApJL, 756, L33

Quinn, S. N., White, R. J., Latham, D. W., et al. 2014, ApJ, 787, 27

Rayner, J. T., Cushing, M. C., \& Vacca, W. D. 2009, ApJS, 185, 289

Rayner, J. T., Toomey, D. W., Onaka, P. M., et al. 2003, PASP, 115, 362 
Rogers, L. A. 2015, ApJ, 801, 41

Rojas-Ayala, B., Covey, K. R., Muirhead, P. S., \& Lloyd, J. P. 2012, ApJ, 748, 93

Sato, B., Izumiura, H., Toyota, E., et al. 2007, ApJ, 661, 527

Schlieder, J. E., Crossfield, I. J. M., Petigura, E. A., et al. 2016, ApJ, 818, 87 Shkolnik, E. L., \& Barman, T. S. 2014, AJ, 148, 64

Southworth, J. 2012, Astrophysics Source Code Library, JKTEBOP: Analyzing light curves of detached eclipsing binaries, ascl:1207.013

Southworth, J., Mancini, L., Maxted, P. F. L., et al. 2012, MNRAS, 422, 3099 Southworth, J., Maxted, P. F. L., \& Smalley, B. 2004, MNRAS, 349, 547

Spurzem, R., Giersz, M., Heggie, D. C., \& Lin, D. N. C. 2009, ApJ, 697, 458

Stauffer, J. R., Rebull, L. M., Bouvier, J., et al. 2016, arXiv:1606.00057

Vacca, W. D., Cushing, M. C., \& Rayner, J. T. 2003, PASP, 115, 389
Vanderburg, A., \& Johnson, J. A. 2014, PASP, 126, 948

van Leeuwen, F. 2009, A\&A, 497, 209

van Saders, J. L., \& Gaudi, B. S. 2011, ApJ, 729, 63

Vogt, S. S., Allen, S. L., Bigelow, B. C., et al. 1994, Proc. SPIE, 2198, 362

Weiss, L. M., \& Marcy, G. W. 2014, ApJL, 783, L6

Weldrake, D. T. F., Sackett, P. D., Bridges, T. J., \& Freeman, K. C. 2005, ApJ, 620,1043

West, A. A., Hawley, S. L., Bochanski, J. J., et al. 2008, AJ, 135, 785

Williams, S. D., Stauffer, J. R., Prosser, C. F., \& Herter, T. 1994, PASP, 106,817

Wolfgang, A., \& Lopez, E. 2015, ApJ, 806, 183

Wolfgang, A., Rogers, L. A., \& Ford, E. B. 2015, arXiv:1504.07557 